DOI: 10.20472/IAC.2018.038.028

IOANA PALADE

Babes-Bolyai University, College for Advenced Performance Studies, Romania

CRISTIAN GAVRILOAIA

Babes-Bolyai University, College for Advanced Performance Studies, Romania

RĂZVAN TIMIS

Babes-Bolyai University, College for Advanced Performance Studies, Romania

\title{
YOU CAN DO IT! A MHEALTH INTERVENTION FOR SMOKING CESSATION
}

\begin{abstract}
:
This paper intends to approach mHealth interventions and their effectiveness when it comes to smoking cessation. Smoking is a problem not only in Romania, but it's a worldwide issue. This topic needs to be further studied because applying technology in medical treatments is not a tackled issue in Romania.

Our paper aims at analyzing the effectiveness of mHealth interventions on smoking cessation, having recourse to several methods: the focus group and the Discrete Choice Experiment. The Discrete Choice Experiment will focus on finding out the factors that drive the individuals to choose an mHealth intervention in order to quit smoking, despite the traditional types of interventions (medical patches, pills). The focus group completes our approach, outlining how individuals perceive the effectiveness of mHealth interventions for smoking cessation and what a mobile app should contain to be effective when ceasing this behavior.

Our findings suggest that individuals tend to turn to a mHealth intervention instead of a traditional one when they can receive rewards or incentives or if they need a quick and accessible solution. What is more, the focus group's results demonstrate the fact that individuals perceive mHealth interventions as not being effective. Also, mobile interventions should be tailored considering the needs of every individual and should contain a section with medical counseling. Considering these results, we believe that the Romanian medical system must introduce mHealth interventions in order to ease individuals' access to health and have a more patient-oriented system.
\end{abstract}

\section{Keywords:}

mHealth, intervention, mobile app, smoking cessation

JEL Classification: 119 